Z Gerontol Geriat 2018 $51: 949$ https://doi.org/10.1007/s00391-018-01466-3 Online publiziert: 19. November 2018

(c) Springer Medizin Verlag GmbH, ein Teil von Springer Nature 2018

CrossMark

\section{Georg Schiffner}

Innere Medizin, Geriatrie und Palliativmedizin, Geriatriezentrum und Palliativbereich, Wilhelmsburger

Krankenhaus Groß-Sand, Hamburg, Deutschland

\section{Originalpublikation}

M. Jamour, B.R. Metz, C. Becker (Hrsg) (2018) Geriatrisch-Rehabilitatives BasisManagement - ein Leitfaden für die Praxis, 1 Aufl. Kohlhammer, Stuttgart $(€ 39,99)$

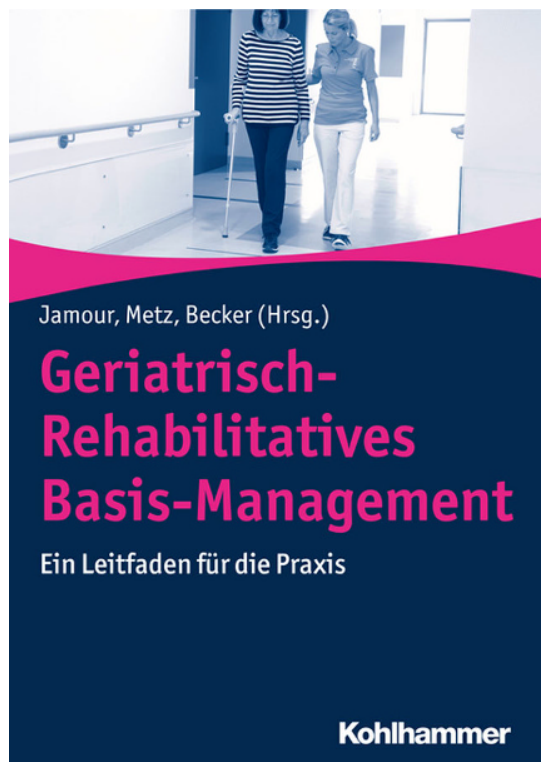

Erstmals ist hiermit ein Kompendium des geriatrisch-rehabilitativen Basismanagements im deutschsprachigen Raum erschienen, welches, gut strukturiert und übersichtlich zusammengefasst, die Bandbreite geriatrischer Aufgabenstellungen in der Rehabilitation beleuchtet. Der selbstgestellte Anspruch, ein geriatrisch-rehabilitatives Basismanagement (GRBM) als „State of the Art" geriatrischer Rehabilitationsmedizin zu veröffentlichen, ist den Autoren in überzeugender Weise gelungen. Mit der Expertise von rund zwei Dutzend erfahrenen Geriatern im Expertenbeirat haben die drei Autoren ein Grundlagenwerk zusammengestellt, welches unabhängig von der Rehabilitationshauptdiagnose Strategien der Rehabilitation multimorbider Patienten praxisnah beschreibt.

Das Buch stellt auf 70 Seiten übersichtlich 12 Module des GRBM dar: Management funktionell relevanter Komorbiditäten der inneren Organe, des Bewegungsapparates, des Nervensystems, der medikamentösen Nonadhärenz, der Polypharmazie, der Rehabilitation bei kognitiven Beeinträchtigungen, Depression und Angststörungen (hier speziell der Sturzangst), der Inkontinenz, Dysphagie und Malnutrition. Vorangestellt findet sich ein Modul $\mathrm{zu}$ "Modulatoren des Reha-Erfolges“, in dem praxisrelevante Aspekte von Assessment, medizinischer Basisdiagnostik und Monitoring, Medikamentenreview und -selbstmanagement sowie Entlass- und Weiterleitungsmanagement beschreiben sind.

Die Autoren unterscheiden zwischen Kernmaßnahmen und bedarfsabhängigen Zusatzmaßnahmen im GRBM. Hierbei beschreiben sie zu den jeweiligen Modulen Indikation bzw. Zielgruppe, Behandlungsziele und -maßnahmen, -frequenz und -dauer, Setting und zeitliche Positionierung im Reha-Verlauf sowie die jeweils beteiligten Berufsgruppen. Unterstützt durch einen gut 40seitigen Anhang gelingt es ihnen, zwischen Darstellung von Evidenzlage und Expertenmeinung einen gut begründeten und doch übersichtlichen Praxisweg zu empfehlen. Hierbei verwenden sie die Nomenklatur deutscher Leitlinien („soll, sollte, kann"), um die Wertigkeit der Empfehlungen aus Expertensicht zu erläutern. Passend dazu wird bereits in der Präambel auf die im Kontext der Multimorbidität bestehende Limitierung eines rein organspezifisch leitliniengerechten Vorgehens hingewiesen, ohne auf den Bezug evidenzbasierter Leitlinien der wissenschaftlichen Fachgesellschaften, insbesondere im Anhang, zu verzichten.

Der vorliegende Praxis-Leitfaden ist für alle Berufsgruppen im geriatrischen Team relevant. Gleichzeitig stellt er Anspruch und Leistungsfähigkeit der geriatrischen Rehabilitation übersichtlich dar, sodass auch andere Leistungserbringer des Gesundheitswesens sowie Politik und Kostenträger genauere Kenntnis erhalten können. Für Mitarbeiter in der Frührehabilitation der Akutgeriatrie zeigt dieser Praxis-Leitfaden einen gelungenen Überblick über die rehabilitativen Aspekte, die das akutmedizinische Denken und Handeln ergänzen. Gleichzeitig verdeutlicht er die unterschiedlichen, sich ergänzenden Schwerpunkte beider geriatrischer Behandlungsformen, was auch im zeitlich unterschiedlichen Einsatz der beschriebenen Maßnahmen zum Ausdruck kommt. Diese Neuerscheinung stellt eine echte Bereicherung unter den geriatrischen Fachbüchern dar.

Somit ist diesem Buch eine weite Verbreitung zu wünschen, sowohl in der geriatrischen Rehabilitation wie in der Akutgeriatrie und darüber hinaus.

\section{Korrespondenzadresse}

\section{Dr. med. Georg Schiffner}

Innere Medizin, Geriatrie und Palliativmedizin, Geriatriezentrum und Palliativbereich, Wilhelmsburger Krankenhaus Groß-Sand Groß-Sand 3, 21107 Hamburg, Deutschland g.schiffner@gross-sand.de

Interessenkonflikt. G. Schiffner gibt an, dass kein Interessenkonflikt besteht. 